\title{
Diversidad de avispas parasitoides (Hymenoptera) en agroecosistemas de palto (Persea americana Mill.) y mandarina (Citrus spp.) en Cañete, Lima, Perú
}

Diversity of parasitoid wasps (Hymenoptera) in avocado (Persea americana Mill.) and tangerine (Citrus spp.) agroecosystems in Cañete, Lima, Peru

Rubén Collantes GonzÁlez ${ }^{1}$ y Alexander Rodríguez Berrio ${ }^{1}$

\section{RESUMEN}

El presente estudio ilustra la diversidad de avispas parasitoides (Hymenoptera), en los agroecosistemas de palto y mandarina en Cañete, Lima (Perú). Se seleccionaron aleatoriamente 48 fincas situadas en los distritos de San Vicente, San Luis, Quilmaná, Nuevo Imperial, Imperial y Lunahuaná, realizándose colecta de especímenes y muestras vegetales con ayuda de red entomológica, viales con etanol al 70\%, bolsas plásticas, cámaras de recuperación de parasitoides, entre otros materiales. Posteriormente, se procedió con la identificación en laboratorio mediante el uso de claves taxonómicas y se desarrolló un análisis clúster jerárquico (método de Ward y distancia euclidiana cuadrada fijada en cinco), para analizar la proximidad entre familias de parasitoides según su importancia ecológica, considerándose como variables para el análisis el número de morfoespecies, plantas asociadas, cantidad de especímenes, localidades de colecta y taxa parasitados. Los resultados obtenidos muestran un total de 92 especímenes colectados, pertenecientes a 10 familias de Hymenoptera parasitoides; destacando Braconidae (14 especies, 28 especímenes) e Ichneumonidae (nueve especies, 38 especímenes), las cuales fueron ubicadas en un mismo conglomerado, debido a que ambos taxa registraron los mayores valores para todas las variables analizadas. Otras familias de parasitoides encontradas fueron Pteromalidae, Mymaridae, Encyrtidae, Eulophidae, Aphelinidae, Chalcididae, Scoliidae y Pompilidae.

Palabras clave: parasitoidismo; parasítica; control biológico; costa peruana; frutales.

\section{ABSTRACT}

The present research illustrates the diversity of parasitic wasps (Hymenoptera), in avocado and tangerine agroecosystems located in Cañete, Lima (Peru). A sample of 48 farms was selected from the districts of San Vicente, San Luis, Quilmana, Nuevo Imperial, Imperial and Lunahuana, to collect wasps and plant samples with an entomological net, vials with etanol $70 \%$, plastic bags, rearing chambers, among other

1 Universidad Nacional Agraria La Molina. Lima, Perú. 
materials. Then, specimens' identification was held in laboratory using taxonomic keys and a hierarchical cluster analysis was developed (Ward Method and Square Euclidean distance fixed in five), to determine the proximity between parasitoid families according to their ecological importance, considering as variables for analysis the number of morph species, associated plants, quantity of specimens, locations and parasited taxa. Results indicate that a total of 92 specimens were collected, belonging to 10 Hymenoptera parasitoids families, from which Braconidae (14 species, 28 specimens) and Ichneumonidae (nine species, 38 specimens), were outstanding and placed in the same cluster because both taxa achieved the major values for all the analyzed variables. Other collected parasitoid families were Pteromalidae, Mymaridae, Encyrtidae, Eulophidae, Aphelinidae, Chalcididae, Scoliidae and Pompilidae.

Key words: Parasitoids; parasitic; biological control; peruvian coast; fruits.

\section{ICHIKLLACHAW}

Kay yachakupanqa willakun Cañete (Lima-Piruu) chakrakunachaw paltu y mandarina murukuykunachaw avispas parasitoideskunapaqmi (Hymenoptera). Tsaypaq akrakashqa chusku chunka puwaq hatun chakrakunata, tsaykuna kaykaayan San Vicente, San Luis, Quilmaná, Mushuq Imperial, Imperial y Lunahuaná distritukunachawmi, tsaykunachawmi ayllpakuyarquu tukuy laaya avispakunata, fruta plantakunatapis red entomológikawan, etanol $70 \%$, plastiku runkukunawan, parasitoidis aylluna kaamarakuna. Tsaykunatam rakiyarquu laburaturyuchaw, kikin pura kastakuna tariyta munar, imanawpis kashqanta rikaykur, maychaw kawayashqanta rikaykur, aykapis kayanqanta rikaykur, mayqan frutakuna palantachaw kawashqanta rikaykur. Tsaychawmi tariyarquu isqun chunka ishkay laayakunata, tsaykuna ayllukaayashqa chunka Hymenoptera parasitoides yawarmayiman; tsaykunachaw Braconidae (chunka chusku laaya, ishkay chunka rikuqkuna), Ichneumonidae (isqun laaya, kima chunka puwaq rikuqkuna) kashqa, paykunam hukllaman churakashqa llapanpita atska kar. Huk yawar mayikuna tarishqa hina Braconidae (chunka chusku laaya, ishkay chunka rikuqkuna), Ichneumonidae (isqun laayakuna, kima chunka puwaq rikuqkuna) kayashqam.

Pushaq shimikuna: parasitoidismu kaq; parasitika kaq; patsachaw kaqwan imatapis hakay; piruupa yunka markankuna; frutaliskuna.

\section{INTRODUCCIÓN}

El Orden Hymenoptera es un taxón complejo y diverso, el cual comprende especies parasitoides que ejercen control biológico sobre plagas, contribuyendo a la sustentabilidad del agroecosistema, pero requiere tiempo y grandes extensiones para establecerse, a diferencia del control químico (Cisneros, 1995, 2012; Hanson y Gauld, 2006; Van Driesche et al., 2007; Altieri y Nicholls, 2010).

Control biológico (CB), es la interacción tritrófica entre planta, plaga y enemigo natural, en la cual intervienen diversos factores y es más compleja al incrementar los componentes, interacciones y su intensidad, lo cual promueve interferencias beneficiosas. Su comprensión requiere conocimiento ecológico y diseño de agroecosistema adecuado, para desarrollar estrategias de CB de conservación (Ugás et al., 2000; Ripa et al., 2008; Altieri y Nicholls, 2010; Quispe, 2012; Garzón et al., 2014). 
Muchos parasitoides completan su dieta con néctar, polen o secreciones de otros insectos, los cuales por su contenido nutricional les sirven para producir huevos y sobrevivir. Las plantas refugio contribuyen al establecimiento de fauna benéfica al ofrecer alimento alternativo y protección durante períodos sensibles del cultivo (Landis et al. 2000; Hanson y Gauld, 2006; Murphy et al., 2006; Van Driesche et al., 2007; Vásquez et al., 2008; Amundson, 2012; Garzón et al., 2014).

La Superfamilia Ichneumonoidea es un taxón importante para Cañete, de lo cual los trabajos de Redolfi y Marín (1992), Velapatiño (1996), Gómez (2010), Huayta (2013), Rodríguez y Gutiérrez (2014), brindan alcances interesantes en cuanto a diversidad y eficacia de CB sobre plagas agrícolas como Spodoptera spp., Pebops ipomoeae, Microthyris anormalis y Ochyrotica fasciata, entre otros.

De acuerdo con MINAGRI (2015), los cultivos de palto (Persea americana Mill.) y mandarina (Citrus spp.), en el Valle de Cañete, ocupan extensiones que superan las 1000 ha y 1500 ha, respectivamente, por lo cual son rubros estratégicos. Adicionalmente, por ser cultivos perennes representan un potencial para el establecimiento de entomofauna benéfica como los parasitoides, siempre y cuando se desarrolle un manejo agronómico responsable y orientado a la conservación de los mismos.

El presente trabajo está orientado a estimar la diversidad de avispas parasitoides en los agroecosistemas de palto y mandarina del Valle de Cañete, Lima, Perú.

\section{MATERIALES Y MÉTODOS}

El área de estudio corresponde al Valle de Cañete y la quebrada de Lunahuaná, Lima, Perú, con altitud variable entre $3-819$ m s. n. m., clima subtropical seco, registrándose para el año 2014 temperatura promedio de $19^{\circ} \mathrm{C}$, máxima de $29^{\circ} \mathrm{C}$ y mínima de $10.1^{\circ}$ C (figura 1); precipitación de $12.4 \mathrm{~mm}_{\text {.año }}{ }^{-1}$ y humedad relativa entre $87-92 \%$ (INDECI, 2002; IRD Costa - UNALM, 2015).Figura 1. Temperatura $\left({ }^{\circ} \mathrm{C}\right)$ en Cañete de enero a diciembre 2014 temperatura promedio de $19^{\circ} \mathrm{C}$, máxima de $29^{\circ} \mathrm{C}$ y mínima de $10.1^{\circ} \mathrm{C}$ (figura 1); precipitación de $12.4 \mathrm{~mm}$.año ${ }^{-1}$ y humedad relativa entre $87-92 \%$ (INDECI, 2002; IRD Costa - UNALM, 2015).

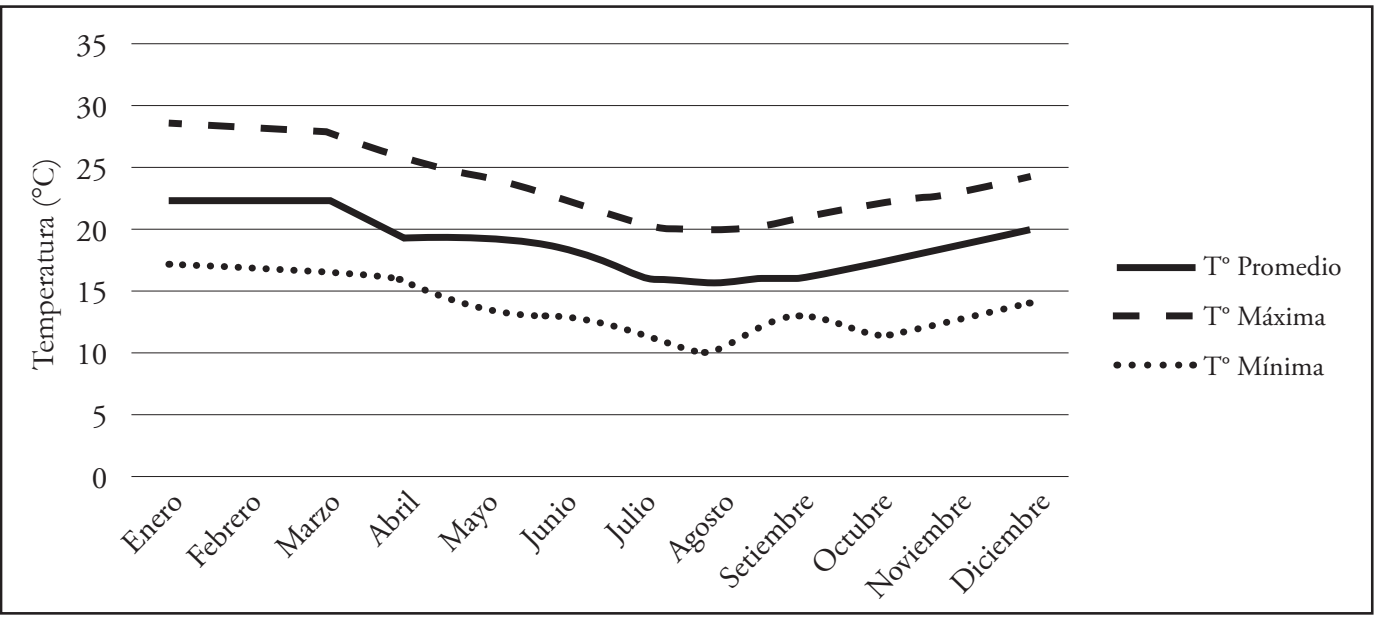

Figura 1. Temperatura $\left({ }^{\circ} \mathrm{C}\right)$ en Cañete de enero a diciembre 2014 
Se visitó fincas productoras de palto y mandarina inscritas en la Asociación de Agricultores de Cañete. De un total de 55 fincas, se obtuvo una muestra irrestricta aleatoria [n $=48]$, mediante el método de proporciones recomendado por Julca et al. (2009). Cada finca visitada fue georreferenciada con GPS, para facilitar su visualización y marcado en Google Earth (figura 2).

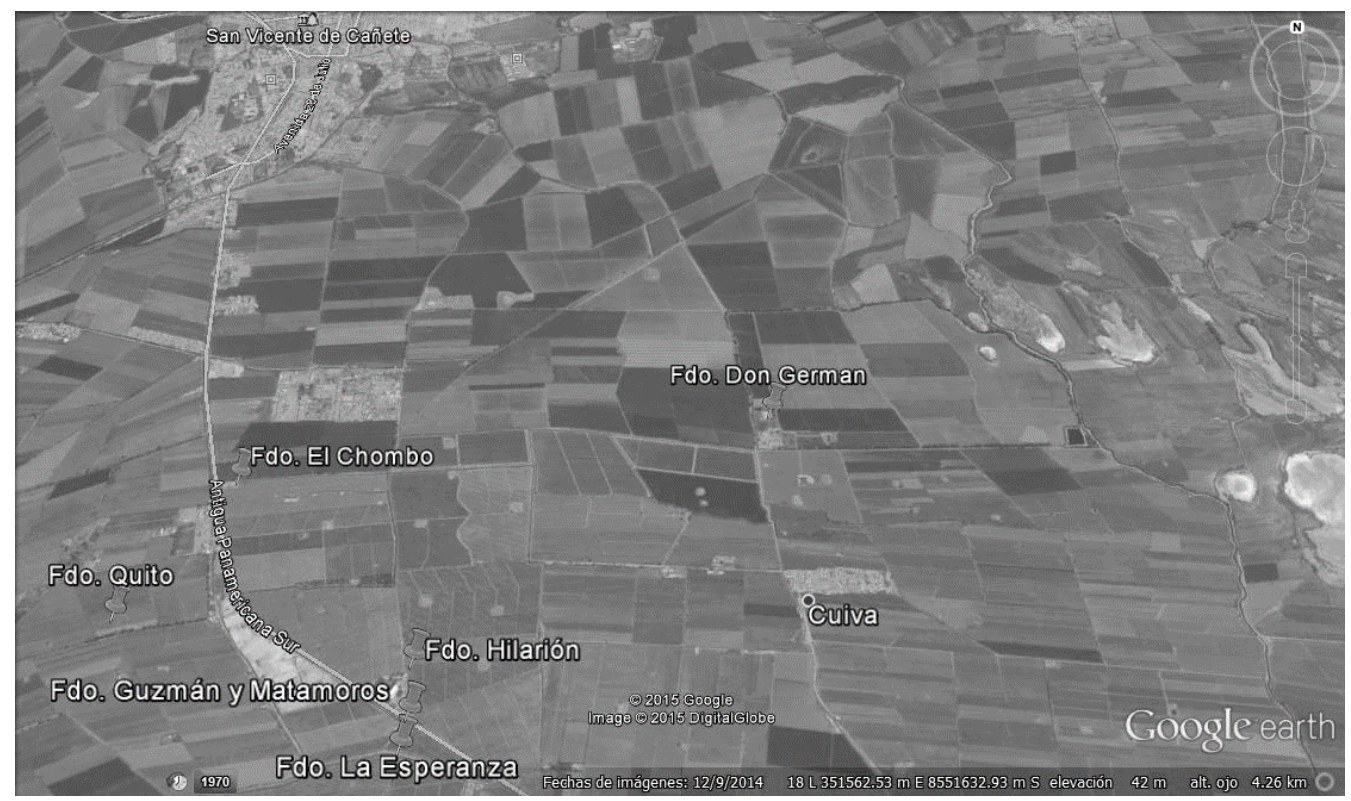

Figura 2. Marcado de fincas visitadas con GPS

El desplazamiento en campo se hizo en motocicleta. Se colectaron avispas parasitoides y material vegetal mediante tijeras, red entomológica, aspirador, viales de vidrio con tapa, etanol al 70\% y bolsas plásticas. Adicionalmente, se emplearon cámaras de recuperación de parasitoides, según la propuesta de Sarmiento y Sánchez (2000).

El acopio de la data se desarrolló principalmente durante la cosecha y la siguiente floración (marzo-agosto, 2015), periodo en el cual las aplicaciones de plaguicidas fueron reducidas. Posteriormente, se procedió con el montaje, rotulado e identificación de especímenes en el Museo de Entomología de la UNALM, consultándose los trabajos de Townes (1969; 1970a; 1970b; 1971), Gauld (1997; 2000) Gauld et al. (2002), Wharton et al. (1998), Fernández y Sharkey (2006), Hanson y Gauld (2006).

\section{RESULTADOS}

El total de especímenes de parasitoides colectados y recuperados fue de 92, procedentes de las localidades de San Luis, San Vicente, Quilmaná, Cuiva y Lunahuaná. En cuanto a la diversidad y abundancia de especies de avispas parasitoides (figura 3), el mayor número de especímenes fue colectado en San Luis (31) y el mayor número de especies se encontró en San Vicente (10); mientras que en Quilmaná hubo un número 
similar de especies respecto a San Luis (siete), pero en número reducido y Lunahuaná fue la que registró los menores valores durante el desarrollo del estudio.

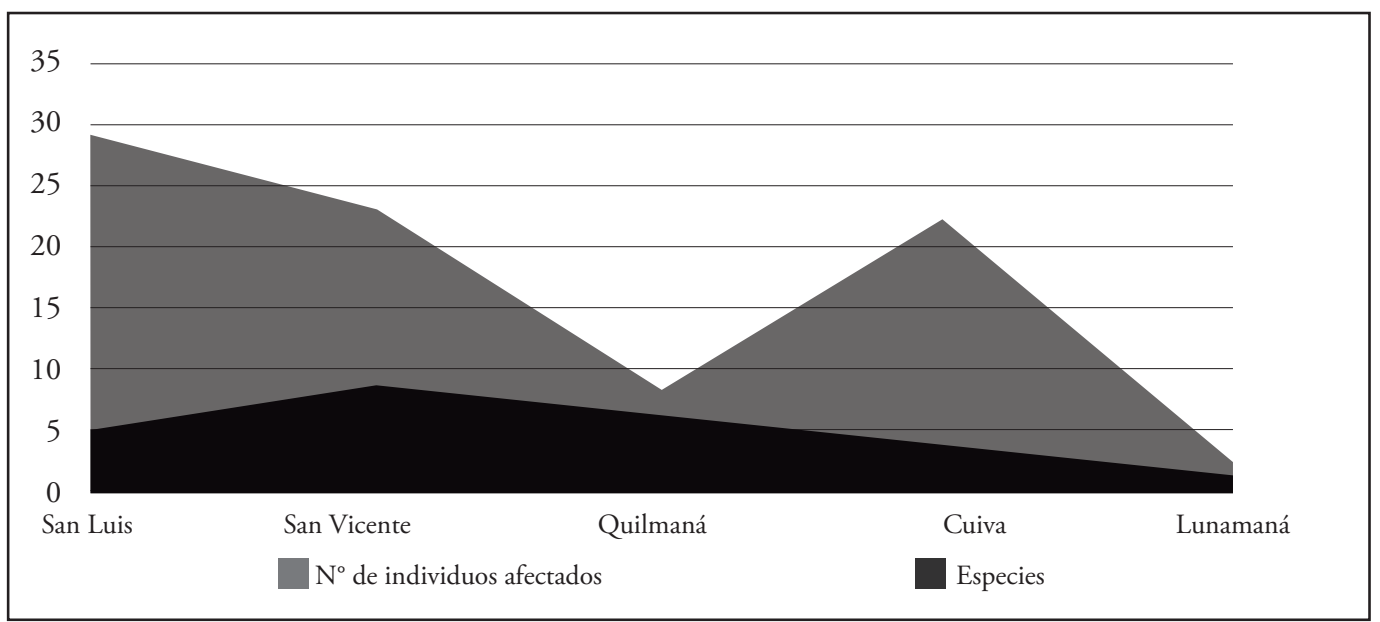

Figura 3. Número de individuos y especies de Hymenoptera parasitoides por localidad en Cañete

Se colectó un total de 10 familias de avispas parasitoides (figura 4), de las cuales Ichneumonidae (41\%) y Braconidae (31\%), fueron las más abundantes y frecuentes. La especie más abundante correspondió a Venturia sp. (figura 5-d), colectada en San Luis (18) y Quilmaná (dos); mientras que Campoletis sp., se colectó en tres localidades (tabla 1).

Otras especies encontradas correspondieron a Chelonus sp. (5-a); Diplazon laetatorius (5b), Anomlaon sinuatum (5-c), Campsomeris sp. (5-e), Pepsis sp. (5-f), Brachymeria sp. (5-g) e individuos de la familia Pteromalidae (5-h). En cuanto a las plantas asociadas, el 56.5\% de los especímenes se colectó en cercos vivos de Acacia horrida (L.) aledaños a campos de mandarina, representados por doce especies en cuatro familias.

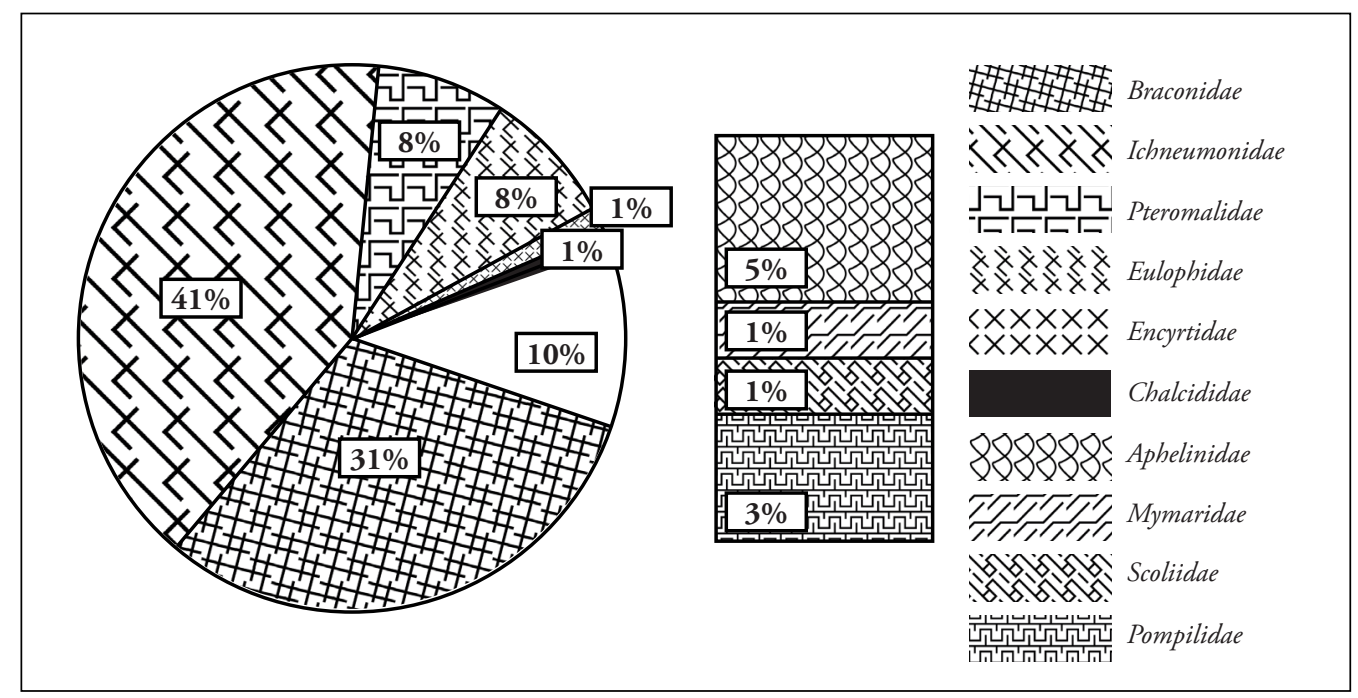

Figura 4. Familias de Hymenoptera parasitoides (\%), en fincas de palto y mandarina, Cañete 
Tabla 1. Especies de Hymenoptera parasitoides colectadas por planta, localidad y presa en Cañete

\begin{tabular}{|c|c|c|c|c|c|}
\hline Familia & Especie & Plantas & $\begin{array}{c}\mathrm{N}^{\circ} \\
\text { individuos }\end{array}$ & Localidad & Presa \\
\hline \multirow{14}{*}{ Ichneumonidae } & \multirow{2}{*}{ Venturia sp. } & Citrus spp. y A. horrida & 18 & San Luis & \multirow{8}{*}{ Lepidóptera } \\
\hline & & P. americana y $A$. horrida & 2 & Quilmaná & \\
\hline & \multirow{3}{*}{ Campoletis sp. } & \multirow{2}{*}{ Citrus spp. y A. borrida } & 2 & San Luis & \\
\hline & & & 2 & San Vicente & \\
\hline & & S. halepense (Citrus spp.) & 1 & Cuiva & \\
\hline & Cremastinae & T. officinale & 2 & Quilmaná & \\
\hline & Campopleginae & Citrus spp. y A. borrida & 1 & San Luis & \\
\hline & Leurus caeruliventris & T. officinale & 1 & Quilmaná & \\
\hline & \multirow{2}{*}{ Diplazon laetatorius } & T. officinale & 1 & Quilmaná & \multirow{2}{*}{ Syrphidae } \\
\hline & & S. halepense (Citrus spp.) & 1 & San Vicente & \\
\hline & \multirow{2}{*}{ Anomalon sinuatum } & $P$. americana y $P$. lucuma & 1 & \multirow{2}{*}{ Quilmaná } & \multirow{3}{*}{$\begin{array}{l}\text { Larvas de } \\
\text { Coleóptera }\end{array}$} \\
\hline & & A. borrida & 1 & & \\
\hline & Anomalon sp. & Citrus spp. y A. borrida & 2 & San Vicente & \\
\hline & Cryptinae & Citrus spp. y $A$. horrida & 3 & San Vicente & $\begin{array}{c}\text { Pupas de } \\
\text { Lepidóptera }\end{array}$ \\
\hline \multirow{20}{*}{ Braconidae } & Chelonus sp. & P. americana y $V$. vinifera & 2 & Lunahuaná & Lepidóptera \\
\hline & \multirow{2}{*}{ Braconinae 1} & T. officinale (Citrus spp.) & 2 & Cuiva & \multirow{11}{*}{$\begin{array}{l}\text { Larvas de } \\
\text { Lepidóptera y } \\
\text { Coleóptera }\end{array}$} \\
\hline & & T. officinale (P. americana) & 1 & Quilmaná & \\
\hline & & Citrus spp. y $A$. borrida & 2 & San Vicente & \\
\hline & Braconinae2 & T. officinale (Citrus spp.) & 1 & Cuiva & \\
\hline & & Amaranthus sp. (Citrus spp.) & 1 & Cuiva & \\
\hline & & Amaranthus sp. (Citrus spp.) & 1 & Cuiva & \\
\hline & Braconinae3 & Citrus spp. y $A$. borrida & 1 & Cuiva & \\
\hline & Braconinge4 & Citrus spp. y A. horrida & 2 & Cuiva & \\
\hline & Braconinae4 & Amaranthus sp. (Citrus spp.) & 1 & Cuiva & \\
\hline & & Citrus spp. y $A$. borrida & 3 & San Vicente & \\
\hline & Braconinaes & Citrus spp. y $A$. borrida & 1 & San Luis & \\
\hline & Braconidae & Citrus spp. & 1 & San Vicente & Lepidóptera \\
\hline & Microgastrinae1 & Citrus spp. y $A$. borrida & 1 & San Vicente & \\
\hline & Microgastrinae2 & T. officinale (Citrus spp.) & 1 & Cuiva & $\begin{array}{l}\text { Larvas de } \\
\text { Lepidóptera }\end{array}$ \\
\hline & Microgastrinae 3 & Citrus spp. & 1 & San Vicente & \\
\hline & Opiinae & Citrus spp. y A. horrida & 2 & San Vicente & Tephritidae \\
\hline & Aphidiinae & T. officinale (P. americana) & 2 & Quilmaná & \\
\hline & Aphidius sp. & S. halepense y T. officinale & 1 & San Vicente & Aphididae \\
\hline & $\begin{array}{l}\text { Lysiphlebus testa- } \\
\text { ceipes }\end{array}$ & S. balepense y T. officinale & 1 & San Vicente & pprocarace \\
\hline Pteromalidae & & Amaranthus sp. (Citrus spp.) & 1 & San Vicente & \\
\hline Pteromalidae & Gen. sp. & Citrus spp. & 6 & San Vicente & P. citrella \\
\hline Mymaridae & Gen. sp. & Citrus spp. & 1 & San Vicente & $\begin{array}{l}\text { Huevos de } \\
\text { Lepidóptera }\end{array}$ \\
\hline Encyrtidae & Gen. sp. & Citrus spp. & 1 & San Vicente & P. citrella \\
\hline
\end{tabular}


Diversidad de avispas parasitoides en agroecosistemas de palto y mandarina en Cañete|

\begin{tabular}{|c|c|c|c|c|c|}
\hline \multirow{3}{*}{ Eulophidae } & \multirow{2}{*}{ Closterocerus sp.? } & \multirow{3}{*}{ Citrus spp. y $A$. borrida } & 1 & San Luis & \multirow{2}{*}{ Hemiptera } \\
\hline & & & 1 & Cuiva & \\
\hline & $\begin{array}{l}\text { Citrostichus phylloc- } \\
\text { nistoides }\end{array}$ & & 5 & San Luis & P. citrella \\
\hline \multirow{2}{*}{ Aphelinidae } & Aphytis lepidosaphes & \multirow{2}{*}{ Citrus spp. } & 3 & \multirow{2}{*}{ San Luis } & \multirow{2}{*}{$\begin{array}{c}\text { Lepidosaphes } \\
\text { beckii }\end{array}$} \\
\hline & Encarsia citrina & & 2 & & \\
\hline Chalcididae & Brachymeria sp. & $P$. americana y $V$. vinifera & 1 & Lunahuaná & Lepidóptera \\
\hline Scoliidae & Campsomeris sp. & P. americana y $P$. lucuma & 1 & Quilmaná & $\begin{array}{c}\text { Larvas de } \\
\text { Scarabaeidae }\end{array}$ \\
\hline \multirow{3}{*}{ Pompilidae } & Tachypompilus sp. & Citrus spp. y $A$. horrida & 1 & \multirow{3}{*}{ Cuiva } & \multirow{3}{*}{ Arañas } \\
\hline & Pepsis sp. & \multirow{2}{*}{ Z. mais y Citrus spp. } & 1 & & \\
\hline & Gen sp. & & 1 & & \\
\hline 10 & 35 & 9 & 92 & 5 & 9 \\
\hline
\end{tabular}

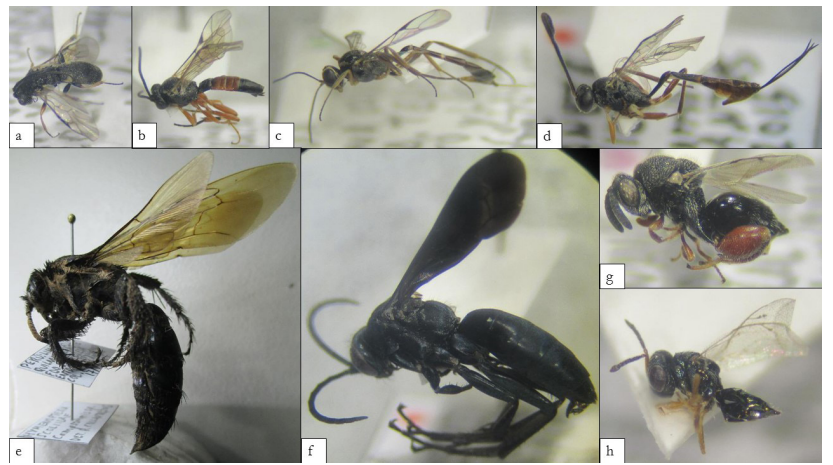

Figura 5. Parasitoides asociados a palto y mandarina en Cañete

El análisis clúster jerárquico (figura 6), indicó que las Familias Braconidae e Ichneumonidae se sitúan en un mismo conglomerado, al haber registrado los mayores valores para morfoespecies colectadas (14; nueve), especies de plantas asociadas (siete; seis), especímenes colectados $(28 ; 38)$, localidades de colecta (cinco; cuatro) y taxa susceptibles de ser parasitados (cuatro; tres).

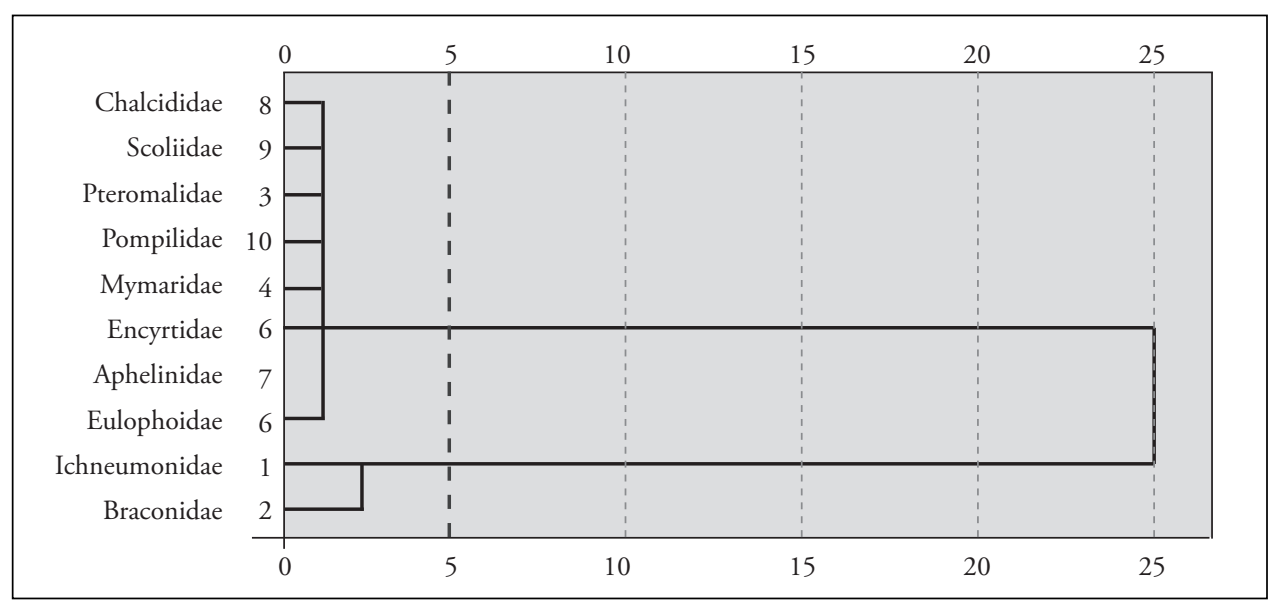

Figura 6. Clúster jerárquico de las familias de Hymenoptera parasitoides colectadas en Cañete 
Se conformaron dos conglomerados:

- Conglomerado 1 (Ichneumonidae y Braconidae): familias que representan más del 20\% de la diversidad tanto de especies como de especímenes colectados, asociadas a más de seis especies vegetales, presentes en todas las localidades de colecta y con capacidad de parasitar al menos tres órdenes de insectos plaga.

- Conglomerado 2 (Eulophidae, Aphelinidae, Encyrtidae, Mymaridae, Pteromalidae, Chalcididae, Scoliidae y Pompilidae): familias que representan menos del 10\% de la diversidad tanto de especies como de especímenes colectados, asociados a no más de tres especies vegetales, presentes en no más de dos localidades de colecta y con capacidad de parasitar no más de dos órdenes de insectos plaga.

\section{DISCUSIÓN}

Entre las razones por las cuales la colecta de especímenes fue limitada, se tienen:

- Manejo agronómico: persisten prácticas convencionales como el uso frecuente de plaguicidas y la quema, las cuales generan perturbaciones en el agroecosistema, comprometiendo así el desarrollo de especies benéficas, tal como señalaron Carlson (1962), Altieri y Nicholls (2010).

- Clima: de febrero a setiembre de 2015 (figura 7), la temperatura promedio fue superior respecto al año anterior, principalmente en los meses de julio a setiembre. Esto pudo repercutir en la actividad de los insectos y la fisiología de los cultivos, observándose en general una floración irregular en campos de cítricos.

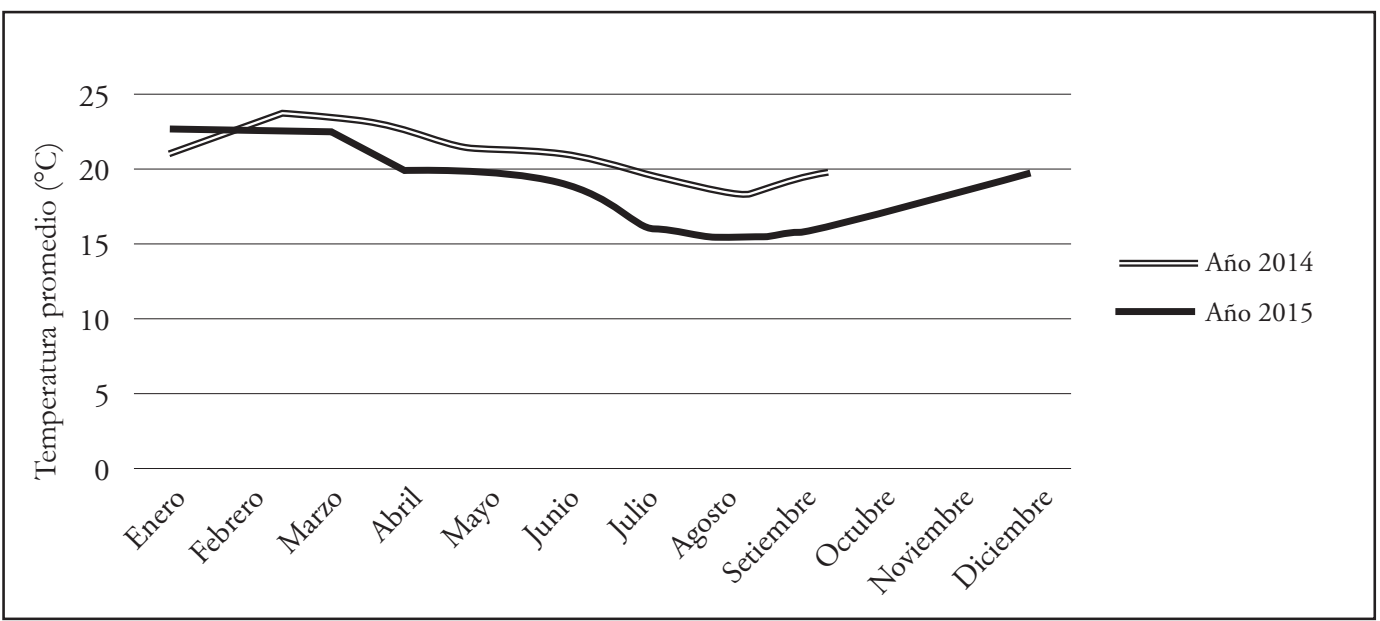

Figura 7. Temperatura promedio mensual $\left({ }^{\circ} \mathrm{C}\right)$ de enero 2014 a setiembre 2015

De acuerdo con Llosa (2014), Perú es el tercer país con mayor riesgo climático, por lo cual la recurrencia de estos fenómenos puede comprometer la seguridad energética y alimentaria. 
- Método de colecta: en estudios desarrollados por Huayta (2013), Rodríguez y Gutiérrez (2014), se empleó trampas, lo cual facilita la obtención de mayor número de especímenes, pero deriva en la pérdida de datos importantes como la fecha y situación de colecta, plantas asociadas, entre otros.

El 72\% de los especímenes colectados en campos de palto y mandarina en Cañete pertenece a la Superfamilia Ichneumonoidea, siendo la especie más colectada Venturia sp. $(22 \%)$. Esto se aproxima a los resultados obtenidos por Gómez (2010), en cultivos de algodonero, camote, maíz, alfalfa y garbanzo en el Valle de Cañete, de los cuales se colectó larvas de Lepidóptera parasitadas, recuperando un 90.59\% de Ichneumonoidea, siendo Campoletis flavicincta la especie predominante (más del 60\%).

Si bien, la Subfamilia Campopleginae fue el taxón más abundante, no se colectó ningún espécimen del género Microcharops, el cual según Rodríguez y Gutiérrez (2014), es abundante en la cuenca del Río Cañete. Estas diferencias en cuanto a predominancia de taxa podrían deberse parcialmente a cambios en el clima y a las características propias de los agroecosistemas estudiados.

Korytkowski (1967) señaló la importancia de Diplazon laetatorius Fabr. (figura 5-b), como parasitoide de larvas Syrphidae (aphidófagos), lo cual se confirmó en campo.

Alvarado et al. (2014) reportaron cuatro especies para Perú del género Anomalon, de las cuales en el presente estudio se encontró $A$. sinuatum (figura 5-c), asociada a palta, lúcuma y acacia en Quilmaná.

La presencia de Chelonus sp. (figura 5) y Campoletis sp., reafirma lo expuesto por Redolfi y Marín (1992), sobre su importancia como parte del complejo de parasitoides de Spodoptera spp., en Cañete. De las 14 subfamilias de Braconidae identificadas por Huayta (2013) en la cuenca del río Lunahuaná, se encontraron Braconinae, Cheloninae, Aphidiinae, Microgastrinae y adicionalmente Opiinae.

La especie vegetal presente en todas las fincas visitadas fue Acacia horrida (L.), la cual constituye un componente fundamental del agroecosistema como cerco vivo, el que ofrece varias de las ventajas señaladas por Garzón et al. (2014).

Si bien el análisis clúster jerárquico situó en un mismo conglomerado a Braconidae e Ichneumonidae por su importancia en la biodiversidad de los agroecosistemas, hay plagas como Phyllocnistis citrella (Stainton, 1856) que cuentan con un complejo de enemigos naturales de las familias Pteromalidae, Encyrtidae y Eulophidae (C. phyllocnistoides), en su mayoría recuperados en laboratorio, siguiendo la metodología sugerida por Sarmiento y Sánchez (2000).

\section{CONCLUSIONES}

La diversidad de Hymenoptera parasitoides asociados a agroecosistemas de palto y mandarina en Cañete, Lima (Perú), está representada por diez familias, destacando Braconidae e Ichneumonidae, situadas en un mismo conglomerado y aportan significativamente a la dimensión ambiental. 
La localidad de San Luis registró el mayor número de especímenes colectados, mientras que en San Vicente se obtuvo el mayor número de especies.

Acacia horrida (L.) es la especie vegetal más común y constituye un corredor biológico potencial.

\section{AGRADECIMIENTOS}

Al Museo de Entomología Dr. Klaus Raven B. de la UNALM, al IRD-Costa, UNALM y al Instituto Valle Grande, por el apoyo logístico. A Percy Peralta, Felizardo Fabián y Elder Tarrillo, por su ayuda durante la fase de campo. Finalmente, a los agricultores por su hospitalidad y colaboración.

\section{REFERENCIAS BIBLIOGRÁFICAS}

Altieri, Miguel y Nicholls, Clara. 2010. Diseños Agroecológicos para Incrementar la Biodiversidad de Entomofauna Benéfica en Agroecosistemas. 1ra. ed. Medellín: Sociedad Científica Latinoamericana de Agroecología (SOCLA).

Alvarado, Mabel; Figueroa, Luis y Rodríguez, Alexander. 2014. «Four species of Anomalon Panzer, 1804 (Hymenoptera: Ichneumonidae) newly recorded for Peru». Rev. Perú. biol. 21(3). 271-274.

Amundson, Susannah. 2012. Cultural Techniques to Improve Yield and Cost Efficiency of Greenhouse Grown Tomatoes. Tesis de Maestría, Universidad de Tennessee, US. Bogotá.

Carson, Rachel. 1962. Silent Spring. Edición del 50 Aniversario. Mariner Books: US.

Cisneros, Fausto. 1995. «Control de Plagas Agrícolas». Control Biológico. 2da. Ed. Cap. 8. Lima: Full Print S.R.L. 102-147.

Cisneros, Fausto. 2012. Control Quimico de Plagas Agrícolas. 1ra ed. Lima: Sociedad Entomológica del Perú.

Fernández, Fernando y Sharkey, Michael. 2006 (eds.). Introducción a los Hymenoptera de la Región Neotropical. Bogotá: Sociedad Colombiana de Entomología y Universidad Nacional de Colombia.

Garzón, Agustín; Bengochea, Paloma y Hiernaux, Luis. 2014. Técnicas y métodos ecológicos de equilibrio entre parásitos, patógenos y cultivos. Ediciones Paraninfo, S.A.

Gauld, Ian. 1997. The Ichneumonidae of Costa Rica, 2. Memoirs of the American Entomological Institute 57. 1-485.

Gauld, Ian. 2000. The Ichneumonidae of Costa Rica, 3. Memoirs of the American Entomological Institute 63. 1-453.

Gauld, Ian; Godoy, Carolina; Sithole, Rudo y Ugalde, Jesús. 2002. The Ichneumonidae of Costa Rica, 4. Memoirs of the American Entomological Institute 66. 1-768. 
Gómez, Isrrael. 2010. Contribución al conocimiento del parasitoidismo de plagas del Orden Lepidóptera en el Valle de Cañete. Tesis para optar el título de Ingeniero Agrónomo, Universidad Nacional Agraria La Molina. Lima: UNALM. 105.

Hanson, Paul y Gauld, Ian (eds.). 2006. Hymenoptera de la Región Neotropical. Memoirs of the American Entomological Institute 77. 1-994.

Huayta, Fredy. 2013. Distribución altitudinal de parasitoides de la Familia Braconidae en la Cuenca del Río Lunahuaná. Tesis para optar el título de Ingeniero Agrónomo, UNALM.

INDECI (Instituto Nacional de Defensa Civil, PE). 2002. Mapa de peligros, Plan de usos del suelo y Propuesta de medidas de mitigación de los efectos producidos por los desastres naturales de las ciudades de la Provincia de Cañete. Lima: Programa de Ciudades Sostenibles, Primera Etapa en colaboración con Universidad Nacional San Luis Gonzaga de Ica.

IRD Costa - Instituto Regional de Desarrollo Costa, Universidad Nacional Agraria La Molina. 2015. Consolidado de la Información Meteorológica del Fundo Don Germán, Cañete. Lima: UNALM.

Julca, Alberto; Rodríguez, Patricia; Meneses, Liliana; Blas, Raúl; Bello, Segundo; Anahui, Juan; Crespo, Reynaldo; Castañeda, Enrique; Reynoso, Auristela; Schuller, Susana; Fundes, Geni y Santibañez, Rubén. 2006. Selección de fuentes naturales para la fertilización de café en el marco de una agricultura orgánica. Lima: Línea base de proyecto financiado por INCAGRO, en alianza con la UNALM, la FDA, la JNC y el INIEA.

Korytkowski, Cheslavo. 1967. Diplazon laetatorius (Fabr.) (Hym.: Ichneumonidae), Ichneumonido syrphidofago poco conocido en el Perú. Rev. Per. Ent. 10(1). 54-58.

Landis, Douglas y otros. 2000. «Habitat Management to enhance Biological Control in IPM». En Kenedy, G. y Sutton, T. (eds.). Emerging technologies for Integrated Pest Management: Concepts, Research and Implementation. Minesota: APS Press. 226-239.

Llosa, Jaime. 2014. Cambio Climático en el Perú. 1 ra. ed. Lima: Fondo Editorial de la Universidad San Ignacio de Loyola.

MINAGRI (Ministerio de Agricultura y Riego). 2015. Consolidado de la Información Agraria de la Provincia de Cañete. Lima: Oficina de Información Agraria, Agencia Agraria de Cañete.

Murphy, Graeme; Ferguson, Gillian y Shipp, Les. 2006. Aphids in greenhouse crops. Fact Sheet, Order N 06-081, 290/621. Ministry of Agriculture, Food and Rural Affairs. Ontario, CAN.

Quispe, Reinaldo. 2012. Áreas de refugio para el mantenimiento de enemigos naturales en el fundo "La Molina» de la Universidad Nacional Agraria La Molina. Tesis para optar el grado de M.Sc. en Entomología. Lima: UNALM. 
Redolfi, Inés y Marin, Rosmarina. 1992. Los controladores biológicos de Spodoptera eridanea (Cramer) en la costa central del Perú. Rev. Per. Ent., 35. 121-124.

Ripa, Renato; Larral, Pilar y Rodríguez, Sharon. 2008. «Control biológico», Cap. 4. En Ripa, Renato y Larral, Pilar (eds.). Manejo de plagas en paltos y cítricos. Lima: Instituto de Investigaciones Agropecuarias, Ministerio de Agricultura. 61-68

Rodríguez, Alexander y Gutiérrez, Silvia. 2014. Diversidad de la Subfamilia Campopleginae-Ichneumonidae (Hymenoptera) en la Cuenca del Río Cañete-Lunabuaná, Perú. Ecología Aplicada 13(2). 147-152.

Sarmiento, Jorge y Sánchez, Guillermo. 2000. Evaluación de insectos. Lima: Departamento de Entomología y Fitopatología, UNALM.

Townes, Henry. 1969. The genera of Ichneumonidae, part 1. Memoirs of the American Entomological Institute 11. 1-300.

Townes, Henry. 1970a. The genera of Ichneumonidae, part 2. Memoirs of the American Entomological Institute 12. 1-537.

Townes, Henry. 1970b. The genera of Ichneumonidae, part 3. Memoirs of the American Entomological Institute 13. 1-307.

Townes, Henry. 1971. The genera of Ichneumonidae, part 4. Memoirs of the American Entomological Institute 17. 1-372.

Ugás, Roberto; Siura, Saray; Delgado de la Flor, Francisco; Casas, Andrés y Toledo, Julio. 2000. Datos Básicos de Hortalizas. Programa de Hortalizas, UNALM.

Van Driesche, Roy; Hoddle, Mark y Center, Ted. 2007. Control de plagas y malezas por enemigos naturales. UDSA. Trad. Ruiz, E, y Coronada, J. México: Universidad de Tamaulipas.

Vásquez, Luis; Matienzo, Yaril; Veitía, Marlene y Alfonso, Janet. 2008. Conservación y manejo de enemigos naturales de insectos fitófagos en los sistemas agrícolas de Cuba. La Habana: INISAV.

Velapatiño, Jorge. 1996. Algunos lepidópteros del camote y sus enemigos naturales en los valles de Cañete y Rímac. Rev. Per. Ent., 39. 111-117.

Wharton, Robert, Marsh, Paul y Sharkey, Michael (eds.). 1998. Manual para los géneros de la Familia Braconidae (Hymenoptera) del Nuevo Mundo. Trad. Imelda Mercado, Yolanda López, Larisa Gálvez y Darío Ramírez. The International Society of Hymenopterists. Washington, D.C.-US.

Fecha de recepción: 05 de noviembre de 2015

Fecha de aceptación: 02 de diciembre de 2015

\section{Correspondencia}

Rubén Collantes González

rdcg31@hotmail.com 